\section{Post-transplant outcome of ovarian tissue cryopreserved after chemotherapy in hematologic malignancies}

Fertility preservation is part of the management of patients with hematologic malignancies because of the gonadotoxicity of treatments such as myeloablative hematopoietic stem cell transplantation conditioning regimens, resulting in premature ovarian failure and infertility.

Ovarian tissue cryopreservation (OTC) has become a fertility preservation technique commonly offered to patients. ${ }^{1}$ The first birth after ovarian tissue transplantation (OTT) into the pelvis was reported in 2004 in a woman previously treated for Hodgkin lymphoma. ${ }^{2}$ So far, more than 130 births have been reported worldwide after autologous $\mathrm{OTT}^{3}$ In some countries OTT is now considered routine. ${ }^{4}$

Ovarian tissue cryopreservation has mostly been performed before exposure to chemotherapy, but the safety regarding ovarian function subsequent to antitumor treatment administration before OTC remains a matter of debate. ${ }^{5}$ The Edinburgh selection criteria for OTC do not consider patients older than 15 years who had previously received chemotherapy. ${ }^{5}$ Indeed, quantitative alterations in the number of oocytes depend on the type of chemotherapy and the age of the patient at the time of treatment. It has been shown that chemotherapy increas- es the rate of nuclear abnormalities in granulosa cells and of vacuolization in oocytes. It also generates vascular alterations and ovarian fibrosis. ${ }^{6,7}$ These abnormalities could not be related to a particular type of chemotherapy.

However, there are many situations in which fertility preservation in patients with hematologic malignancies cannot be performed before the initiation of the chemotherapy. These include patients with severe neutropenia and/or coagulation disorders at diagnosis, patients requiring immediate treatment or patients initially treated with chemotherapy with low gonadotoxic risk in whom a relapse or progression calls for some other chemotherapy with a higher gonadotoxic risk. ${ }^{8}$ Restoration of ovarian function after transplantation of ovarian tissue cryopreserved after the beginning of chemotherapy has been reported in a few cases. The first birth after transplantation of ovarian tissue previously exposed to chemotherapy was reported in $2005 .{ }^{9}$ In 2016 , the same team reported on ten patients previously exposed of whom $40 \%$ had had at least one child after OTT with a mean follow up of 3.18 years. ${ }^{10}$ Determining in a large series of patients whether chemotherapy received before OTC could have a negative impact on ovarian function after transplantation of ovarian tissue remains a major issue.

We report here on 25 patients who had been treated by chemotherapy for hematologic malignancies before OTC, who wished to become pregnant after the cure of
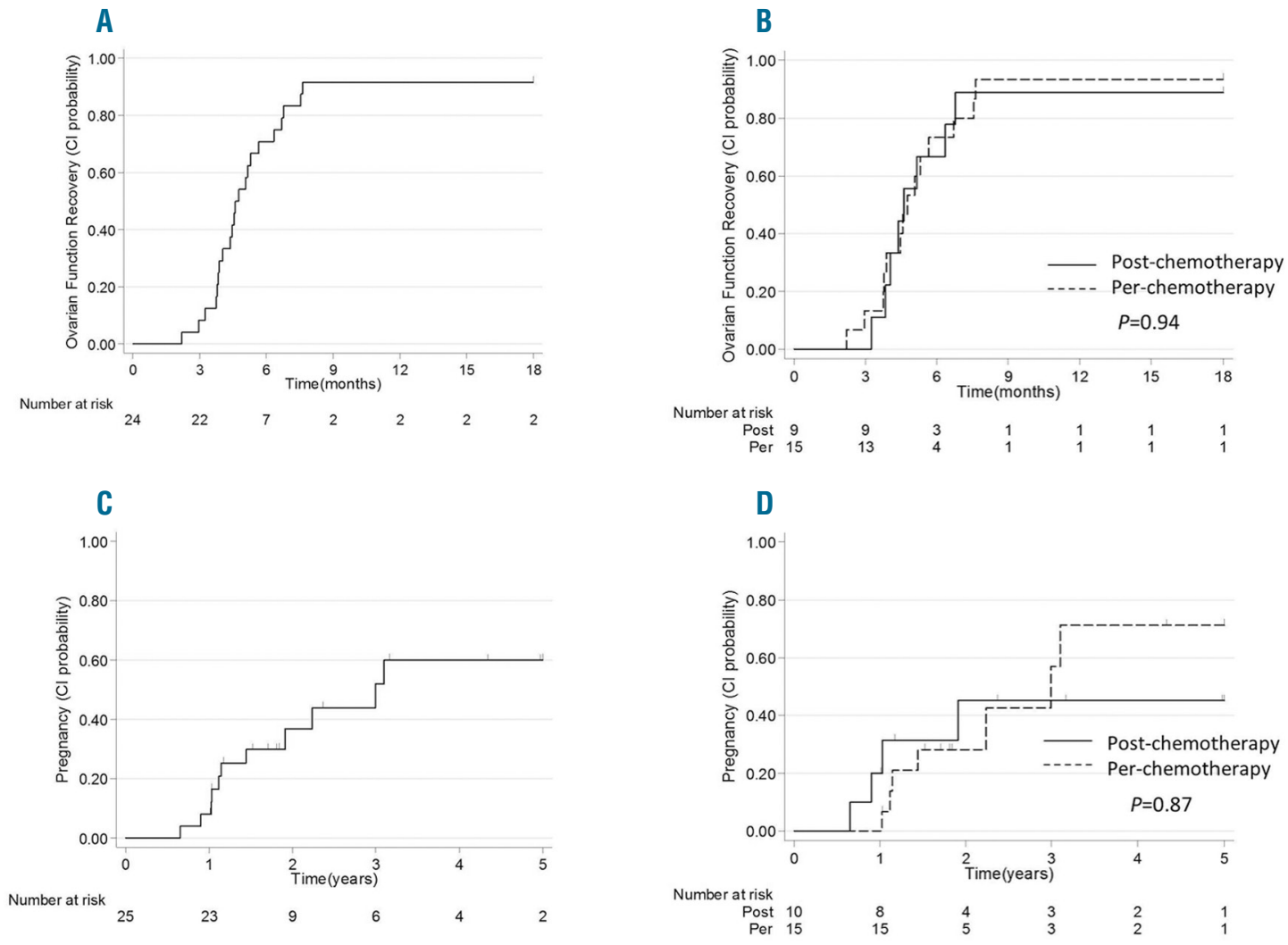

Figure 1. Ovarian function recovery and pregnancy after ovarian tissue transplantation. Cumulative incidence of ovarian function recovery related to total population (A) and if ovarian tissue cryopreservation was carried out post- or per-chemotherapy (B). Cumulative incidence of pregnancy related to total population (C) and if ovarian tissue cryopreservation was carried out post- or per-chemotherapy (D). 
their disease, and who underwent an orthotopic OTT. Our study demonstrates the efficacy of OTT in this setting.

Our OTT program had been approved by the ethical committee of the Pitié-Salpêtrière Hospital. Appropriate consent forms were signed by all patients. Among these 25 patients, 16 were diagnosed with Hodgkin lymphoma, 7 with non-Hodgkin disease, and 2 with acute myeloid leukemia (AML) (patient characteristics are summarized in Table 1).

Ovarian tissue cryopreservation was planned as soon as a highly gonadotoxic treatment was decided. Cryopreservation was performed after the patient reached complete remission to reduce any intra-ovarian tumor infiltration. The ovarian tissue was collected under laparoscopy. Once the ovarian tissue had been removed,

Table 1. Characteristics of patients at the time of ovarian tissue cryopreservation, treatments received before and after ovarian tissue cryopreservation and outcomes of ovarian tissue transplantation.

\begin{tabular}{|c|c|c|c|c|c|c|}
\hline \multirow[t]{2}{*}{ Patient $n$. } & \multirow[t]{2}{*}{ Pathology } & \multicolumn{2}{|c|}{ Age (vears) } & \multirow{2}{*}{$\begin{array}{c}\text { Treatment received } \\
\text { before OTC }\end{array}$} & \multirow{2}{*}{$\begin{array}{c}\text { Treatment received } \\
\text { after OTC }\end{array}$} & \multirow{2}{*}{$\begin{array}{c}\text { OII } \\
\text { outcome }\end{array}$} \\
\hline & & OTC & OाT & & & \\
\hline \multicolumn{7}{|c|}{ POST-CHEMOTHERAPY } \\
\hline 1 & HL & 27.1 & 37.2 & $6 \times$ VEBED & Autologous HSCT & 1 birth \\
\hline 2 & HL & 24.4 & 31.4 & $3 \times \mathrm{ABVD}$ & $\begin{array}{c}\text { Autologous and allogeneic HSCT } \\
\text { (TBI 2Gy) }\end{array}$ & 1 birth \\
\hline 3 & HL & 27.8 & 37.8 & $6 \times \mathrm{ABVD}$ & Autologous HSCT (TBI 6 Gy) & OFR \\
\hline 4 & HL & 31.6 & 36.5 & $6 \mathrm{x}$ EBVP & Autologous HSCT & $\begin{array}{l}1 \text { biochemical } \\
\text { pregnancy, } \\
1 \text { birth }\end{array}$ \\
\hline 5 & HL & 27.0 & 32.2 & $6 \times \mathrm{ABVD}$ & Autologous HSCT & 1 birth \\
\hline 6 & HL & 30.9 & 37.7 & $2 \times \mathrm{ABVD}, 4 \times \mathrm{BEACOPP}$ & Autologous HSCT & OFR \\
\hline 7 & HL & 28.1 & 32.8 & $\begin{array}{c}4 \text { x BEACOPP, } \\
4 \text { x BEACOPPesc, } \\
3 \text { x DHAP, } \\
\text { Autologous HSCT** }\end{array}$ & Allogeneic HSCT (TBI 2Gy) & OFR \\
\hline 8 & HL & 19.9 & 24.1 & $8 \times$ EBVP, $3 \times$ MOPP & Autologous HSCT (TBI*) & OFR \\
\hline 9 & HL & 18.8 & 29.7 & $6 \times \mathrm{ABVD}$ & Autologous HSCT & NOFR \\
\hline 10 & NHD & 26.4 & 34.4 & 7 x R-CHOP, Autologous HSCT** & $2^{\text {nd }}$ Autologous HSCT & OFR \\
\hline \multicolumn{7}{|c|}{ PER-CHEMOTHERAPY } \\
\hline 11 & HL & 18.6 & 24.6 & $2 \times$ VBVP, $2 \times$ MOPP, $2 \times$ OPPA & Autologous HSCT & 2 births, 1 miscarriage \\
\hline 12 & HL & 30.7 & 33.9 & $2 \times$ ABVD, 1 x BEACOPPesc & Autologous HSCT & OFR \\
\hline 13 & HL & 18.9 & 26.7 & ABVD & Autologous HSCT & OFR \\
\hline 14 & HL & 23.8 & 31.4 & $6+4 \times \mathrm{ABVD}$ & Autologous HSCT & 1 birth \\
\hline 15 & HL & 31.7 & 37.7 & $4 \times \mathrm{ABVD}, 2 \times \mathrm{MINE}$ & Autologous and allogeneic HSCT & OFR \\
\hline 16 & HL & 21.0 & 28.6 & $4 \times \mathrm{ABVD}$ & Autologous HSCT & $\begin{array}{c}1 \text { miscarriage after ART, } \\
1 \text { ongoing pregnancy } \\
\text { after ART }\end{array}$ \\
\hline 17 & HL & 27.9 & 35.5 & $4 \times \mathrm{ABVD}$ & Autologous HSCT (TBI 10Gy) & $\begin{array}{l}1 \text { abortion, } 1 \text { birth, } \\
1 \text { miscarriage }\end{array}$ \\
\hline 18 & NHD & 21.3 & 27.2 & $1 \times \mathrm{CHOP}$ & Allogeneic HSCT & 1 ongoing pregnancy \\
\hline 19 & NHD & 31.9 & 34.4 & $6 \times$ R-CHOP, $3 \times$ R-ESHAP & Autologous HSCT & NOFR \\
\hline 20 & NHD & 29.8 & 40.4 & 4 x ACVBP & Autologous HSCT & OFR \\
\hline 21 & NHD & 16.6 & 28.7 & $\begin{array}{l}\text { High-dose chemotherapy } \\
\text { including cyclophosphamide x2 } \\
\text { several Vinblastine infusions }\end{array}$ & $\begin{array}{c}\text { Autologous and } \\
\text { allogeneic HSCT (TBI*) }\end{array}$ & 1 birth \\
\hline 22 & NHD & 25.9 & 29.0 & $1 \times \mathrm{ABVD}$ & Autologous HSCT & $\begin{array}{c}2 \text { miscarriages, } 1 \text { birth, } \\
1 \text { ongoing pregnancy }\end{array}$ \\
\hline 23 & NHD & 29.5 & 31.3 & $1 \times$ ACVBP & Autologous and allogeneic HSCT & OFR \\
\hline 24 & AML & 26.5 & 37.2 & $2 \times \mathrm{ADE}$ & Allogeneic HSCT (TBI*) & OFR \\
\hline 25 & AML & 19.0 & 27.7 & $1 \times$ MAC, $1 \times$ HDAC+ASPA & Allogeneic HSCT & OFR \\
\hline
\end{tabular}

ART: assisted reproductive technology; biochemical pregnancy: $\beta$-hCG under $100 \mathrm{mIU} / \mathrm{mL}$ and no sign of clinical pregnancy; HL: Hodgkin lymphoma; HSCT: hematopoietic stem cell transplantation; NHD: non-Hodgkin disease; NOFR: no ovarian function recovery; OFR: ovarian function recovery; OTC: ovarian tissue cryopreservation; OTT: ovarian tissue transplantation; TBI: total body irradiation. *Unknown dose. **Despite gonadotoxic treatment before OTC, residual ovarian function was considered compatible with fertility preservation. Treatments: ABVD: doxorubicin, bleomycin, vinblastine, dacarbazine; ACVBP: doxorubicin, cyclophosphamide, vindesine, bleomycin, prednisone; ADE: daunorubicine, aracytine, etoposide; BEACOPP and BEACOPPesc: bleomycin, etoposide, doxorubicin, cyclophosphamide, vincristine, procarbazine, prednisone; EBVP: epirubicin, bleomycin, vinblastine, prednisone; HDAC+ASPA: aracytine; L-aspariginase; MAC: aracytine, mitoxantrone; MINE: mitoguazone, ifosfamide, navelbine, etoposide; MOPP: mechlorethamine, vincristine, procarbazine, prednisone; OPPA: vincristine, prednisone, procarbazine, doxorubicine; R-CHOP: rituximab, doxorubicin, cyclophosphamide, vincristine, prednisone; R-ESHAP: rituximab, cisplatin, etoposide, cytarabine, methylprednisolone; VBVP: vinblastine, bleomycin, etoposide, prednisone; VEBED: vinblastine, epirubicin, bleomycin, etoposide, prednisone. 
it was placed in a sterile transport medium (FerticultTM HEPES, Fertipro, Beernem, Belgium) and transported on ice to the laboratory for freezing and cryopreservation. Most of the ovarian medulla tissue was removed and the ovarian cortex was split into fragments. Each fragment was placed into a cryoval containing $1 \mathrm{~mL}$ of freezing solution. Dimethylsulfoxide (Braun Medical SA, Boulogne-Billancourt, France or WAK-Chemie Medical $\mathrm{GmbH}$, Steinbach, Germany) was used as the cryoprotectant and a slow cooling protocol was implemented.

At the time of OTC, the patients were either some time (> 3 months) from their last chemotherapy ("postchemotherapy" group, $\mathrm{n}=10,40 \%$ ) and ovarian sampling was carried out before resuming chemotherapy, or were undergoing chemotherapy ("per-chemotherapy" group, $n=15,60 \%$ ) for progression of the disease or for emergency treatment. No per-chemotherapy patient received LHRH analogs before or per OTC. This information was not recorded for post-chemotherapy patients. Indication of ovarian cryopreservation was autologous $(n=17)$, allogeneic $(n=4)$, or autologous and allogeneic hematopoietic stem cell transplantation $(n=4)$. In seven patients, the conditioning regimen included total body irradiation (TBI).

All OTT were carried out after approval from the hematologist and after checking the absence of tumor contamination in a fragment of ovarian cortex by histological study. For the two patients who had AML and were in complete remission at the time of OTC, a negative minimal residual disease (MRD) was assessed by molecular biology in one of the randomly selected frozen ovarian fragments (MLL-AF4 and RUNX1 mutation as MRD targets).

On the day of OTT, the ovary fragments were thawed according to a rapid thawing protocol and placed in successive baths of thawing solution, with decreasing concentrations of dimethylsulfoxide. The ovarian tissue was transplanted by laparoscopy. The fragments were grafted orthotopically in two sub-peritoneal pouches created in the ovarian fossa. Thawed ovarian fragments were attached to one another and placed in the transplant sites. After OTT, the ovarian function was monitored each month by measuring FSH, $\mathrm{LH}$, estradiol and $\mathrm{AMH}$ to assess the recovery of normal levels of gonadotropins and renewed secretion of estradiol by the transplant, and by trans-vaginal ultrasound to assess follicular growth in the ovarian transplants. The criterion used to define complete recovery of ovarian function was the occurrence of menstruation. Pregnancy was assessed by $\beta$-hCG blood test.

Cumulative incidence of ovarian function recovery (OFR) was defined as the time between transplantation and menstruation recovery, censoring patients with no recovery at the last follow up. The cumulative incidence of pregnancy was measured by the time between transplantation and first pregnancy, censoring patients who were not pregnant at the last follow up. Comparisons between the two subgroups were performed with an univariate Cox model (STATA 12.0 Corporation, College Station, TX, USA).

The median age of patients at the time of OTC in our cohort was 26.5 years (range, 16.6-31.9), and was similar to that in previously reported cohorts. ${ }^{10,11}$ There was no difference in median age between the post-chemotherapy group (27.1 years; range, 18.8-31.6) and the perchemotherapy group (25.9 years; range, 16.6-31) $(P=0.62)$. The median time between OTC and OTT was 6.8 years. The median follow up after ovarian transplantation was 32 months.
At the time of OTT, all patients except one were under premature ovarian failure. This particular patient was not included in the cumulative incidence evaluation of ovarian function recovery. The cumulative incidence of ovarian function recovery at one year was 92\% (95\% CI: 77\% to $99 \%$ ) with a median time to recovery of 4.6 months (range, 2.2-7.6). No difference was found between postchemotherapy patients [cumulative incidence $89 \%$ (95\%CI: 61-99\%)] and per-chemotherapy patients [cumulative incidence 93\% (95\%CI: 74-99\%)] $(P=0.94)$ (Figure $1 \mathrm{~A}$ and $\mathrm{B}$ ). These results compared favorably with those reported in patients who had not received chemotherapy before ovarian cryopreservation, showing a 1-year recovery of ovarian function in $67 \%$ of patients. ${ }^{11}$

In our study, the cumulative incidence of pregnancy was $52 \%(95 \% \mathrm{CI}: 31-77 \%)$ at three years and $60 \%$ (95\% CI: $37-83 \%$ ) at five years, with no significant difference between post and per-chemotherapy groups [3-year cumulative incidence of pregnancy: $45 \%$ (95\%CI: 19 $81 \%$ ) vs. $57 \%$ (95\% CI: $28-88 \%)$, respectively, $(P=0.87)$ ] (Figure $1 \mathrm{C}$ and $\mathrm{D})$. In the whole cohort, 11 patients became pregnant at least once $(41 \%$ of all patients and $46 \%$ of patients who recovered ovarian function) and gave birth to at least one healthy child $(n=8)$ or had an ongoing pregnancy $(n=3)$. These pregnancy rates are similar to those published by Meirow et al. $(40 \%)$ in their series of ten patients exposed to chemotherapy prior to ovarian preservation. ${ }^{10}$ Interestingly, the rate of women who became pregnant at least once was $32.6 \%$ in the series of 49 patients who had an ovarian tissue cryopreservation before chemotherapy. ${ }^{11}$

To our knowledge, this study is the largest of its kind and suggests that the results of OTC are not affected by whether the patient receives or does not receive chemotherapy prior to cryopreservation. Moreover, from a practical point of view, a recent exposure to chemotherapy ( $<3$ months) does not modify the chances of recovering ovarian function and becoming pregnant.

In conclusion, in malignant hematologic diseases where starting chemotherapy may compete with early fertility preservation, ovarian tissue cryopreservation performed at any time, including after the start of chemotherapy, appears efficient and should be the preferred technique. The alternative (cryopreservation of mature oocytes after initiation of chemotherapy) is really not very efficacious. ${ }^{12}$ To preserve fertility even after initiation of chemotherapy may allow a larger number of patients to become candidates for OCT and thus maintain their chances of motherhood. This is indeed a major factor that improves the quality of life of cancer survivors. ${ }^{13}$ Our results may be regarded as a very important part of the information given to the patients with hematologic malignancies.

Catherine Poirot, ${ }^{1,2}$ Anne Fortin, ${ }^{3}$ Nathalie Dhédin, ${ }^{1}$ Pauline Brice, ${ }^{4}$ Gérard Socié, 5,6 Jean-Marc Lacorte, ${ }^{2,7}$ Jean-Paul Akakpo, ${ }^{8}$ Catherine Genestie," Jean-Paul Vernant, ${ }^{2,10}$ Thierry Leblanc, ${ }^{11}$ Jean Gabarre, ${ }^{10}$ Alain Delmer, ${ }^{12,13}$ Yasmina Badachi, ${ }^{8}$ Véronique Drouineaud, ${ }^{14}$ Céline Chalas, ${ }^{14}$ Sophie Egels, ${ }^{8}$ Philippe Touraine, ${ }^{2,15}$ Marc Dommergues, ${ }^{3,2}$ Géraldine Lebègue, ${ }^{3}$ Jean-Philippe Wolf, ${ }^{14,16}$ Frédérique Capron, ${ }^{9,2}$ Gilles Lefebvre, ${ }^{3}$ and Nicolas Boissel,17

${ }^{1}$ Department of Hematology, AYA Unit, AP-HP, Saint-Louis Hospital, Paris; ${ }^{2}$ Sorbonne University, Paris; ${ }^{3}$ Department of Obstetrics and Gynecology, AP-HP, Pitié-Salpêtrière-Charles Foix University Hospital, Paris; ${ }^{4}$ Department of Hematology-Oncology, AP-HP, SaintLouis Hospital, Paris; ${ }^{5}$ Department of Hematology-BMT, AP-HP, 
Saint-Louis Hospital, Paris; 'University Paris 7, and Inserm UMR 1160, Paris; 'Department of Hormonal Biochemistry, AP-HP, PitiéSalpetrière-Charles Foix University Hospital, Paris; ${ }^{8}$ Department of Radiology, AP-HP, Pitie-Salpêtrière-Charles Foix University Hospital, Paris; 'Department of Pathology, AP-HP, Pitié-Salpêtrière-Charles Foix University Hospital, Paris; ${ }^{10}$ Department of Hematology, AP-HP, Pitié-Salpêtrière-Charles Foix University Hospital, Paris; ${ }^{1}$ Department of Pediatric Hematology, AP-HP, Robert-Debré University Hospital, Paris; ${ }^{2}$ Department of Clinical Hematology, Reims University Hospital, Reims; ${ }^{13}$ Reims Champagne-Ardenne University, Reims; ${ }^{14}$ Department of Reproductive Biology, AP-HP, Cochin Hospital, Paris; ${ }^{15}$ Department of Endocrinology, AP-HP, Pitié-Salpêtrière-Charles Foix University Hospital, Paris; ${ }^{16}$ Descartes University, Paris and ${ }^{17}$ Diderot University, EA3518, Paris, France.

Acknowledgments: we would like to thank the hematologists who referred their patients to us: Lionel Ades, André Baruchel, Agnès Buzyn, Patrice Carde, Sylvain Choquet, Serge Cremades, Bernard Desablens, Hélène Esperou, Jamile Frayfer, Kamel Ghomari, Hugo Gonzales, Quoc Hung Le, Stéphanie N'Guyen, Regis Peffault de Latour, Marie Dominique Tabone, Aliénor Xhaard, Jean Marc Zini. We would like to thank Christophe Marzac and Elizabeth Macintyre for determining minimal residual disease in ovarian cortex. We thank Sophie Bringer, Béatrice Delépine, Hélène Letur, Reza Majidi, Emmanuelle Mathieu, Annick Neuraz, Bénédicte Paillusson, Lise Selleret, and Antoine Torre for their contributions to the care of the patients after ovarian tissue transplantation. We thank Hervé Oya for data management, and Alain Mallet, Merry Mazmanian and Cécile Kedzia, project managers, Corinne Journo and Lilia Grira for their technical assistance.

Funding: this study was supported by the "Agence de la Biomédecine" (France's biomedical office).

Correspondence: CATHERINE POIROT. catherine.poirot@aphp.fr doi:10.3324/haematol.2018.211094

Information on authorship, contributions, and financial \& other disclosures was provided by the authors and is available with the online version of this article at www. haematologica.org.

\section{References}

1. Oktay K, Harvey BE, Partridge AH, et al. Fertility preservation in patients with cancer: ASCO clinical practice guideline update. J Clin Oncol. 2018;36(19):1994-2001.

2. Donnez J, Dolmans MM, Demylle D, et al. Livebirth after orthotopic transplantation of cryopreserved ovarian tissue. Lancet. 2004; 363(9443):1405-1410.

3. Donnez J, Dolmans MM. Fertility preservation in women. N Engl J Med. 2017;377(17):1657-1665.

4. Gellert SE, Pors SE, Kristensen SG, Bay-Bjorn AM, Ernst E, Andersen CY. Transplantation of frozen-thawed ovarian tissue: an update on worldwide activity published in peer-reviewed papers and on the Danish cohort. J Assist Reprod Genet. 2018;35(4):561-570.

5. Anderson RA, Mitchell RT, Kelsey TW, et al. Cancer treatment and gonadal function: experimental and established strategies for fertility preservation in children and young adults. Lancet Diabetes Endocrinol. 2015;3(7):556-567.

6. Abir R, Ben-Haroush A, Felz C, et al. Selection of patients before and after anticancer treatment for ovarian cryopreservation. Hum Reprod. 2008;23(4):869-877.

7. Meirow D, Dor J, Kaufman B, et al. Cortical fibrosis and blood-vessels damage in human ovaries exposed to chemotherapy. Potential mechanisms of ovarian injury. Hum Reprod. 2007;22(6):1626-1633.

8. Noetzli J, Voruz S, Wunder D, et al. Ten year Single-center experience in fertility preservation of 459 patients suffering from acute leukaemia. Blood. 2015;126(23):3751.

9. Meirow D, Levron J, Eldar-Geva T, et al. Pregnancy after transplantation of cryopreserved ovarian tissue in patient with ovarian failure after chemotherapy. N Engl J Med. 2005;353(3):318-321.

10. Meirow D, Ra'anani H, Shapira M, et al. Transplantations of frozen thawed ovarian tissue demonstrate high reproductive performance and the need to revise restrictive criteria. Fertil Steril. 2016;106(2):467-474.

11. Van der Ven H, Liebenthron J, Beckmann M, et al. Ninety-five orthotopic transplantations in 74 women of ovarian tissue after cytotoxic treatment in a fertility preservation network: tissue activity, pregnancy and delivery rates. Hum Reprod. 2016;31(9):2031-2041.

12. Dolmans MM, Demylle D, Martinez-Madrid B, et al. Efficacy of in vitro fertilization after chemotherapy. Fertil Steril. 2005;83(4):897901.

13. Schover LR, Rybicki LA, Martin BA, Bringelsen KA. Having children after cancer. A pilot survey of survivors' attitudes and experiences. Cancer. 1999;86(4):697-709. 